\title{
BARYONIC SYSTEMS WITH CHARM AND BOTTOM IN THE BOUND STATE SOLITON MODEL 1
}

\author{
V.B.Kopeliovich \\ Institute for Nuclear Research of the Russian Academy of Sciences, \\ 60th October Anniversary Prospect 7A, Moscow 117312
}

\begin{abstract}
The binding energies of baryonic systems with baryon number $B=2,3$ and 4 possessing heavy flavor, charm or bottom, are estimated within the rigid oscillator version of the bound state approach to chiral soliton models. Two tendencies are noted: the binding energy increases with increasing mass of the flavor and with increasing $B$. Therefore, the charmed or bottomed baryonic systems have more chances to be bound than strange baryonic systems discussed previously. The flavor symmetry breaking in decay constants $F$ is considered which is especially important for baryonic systems with bottom quantum number.
\end{abstract}

\section{Introduction}

Many efforts have been done lately to investigate the properties of baryonic systems $(B S)$ with nonzero strangeness, first of all the possibility of the existence of states stable relative to strong decays.

Recently some of the predictions of theory began to find experimental confirmation. The near-threshold enhancement in $\Lambda \Lambda$ system observed in [1] can be interpreted as a component of 27-plet obtained from the bound $S U(2)$ torus-like configuration with $B=2$ by means of collective coordinates method described in [2, 3]. Similar enhancement in $\Lambda N$ system has been observed many years ago in the kaon production reaction on nucleons [4] and confirmed also in $\Lambda p$ scattering [5]. It can belong to 27-plet or to antidecuplet of dibaryons. The singlet $N N$ scattering state with isospin $T=1$ belongs to the 27-plet (for review of theoretical predictions in $B=2$ sector see, e.g. [6]). Analogous results are obtained in more conventional potential approach as well.

The question if the $B S$ with flavor different from $u$ and $d$ can exist, is more general, of course. Charm, bottom or top quantum numbers are also of interest. Their consideration can be performed in the framework of chiral soliton models, in particular, the bound state approach to heavy flavors proposed in [7] and developed in [8]-[10]. Although charmed and bottomed $B S$ have less chances to play some important role in astrophysics than the strange ones (it is not excluded, however!) their studies can be very useful for understanding of the peculiarities of nuclear matter fragments with unusual properties. It might be similar to heavy quarkonia which studies were very important for development and checking of $Q C D$ itself.

Here the baryonic systems with heavy flavors are considered within the rigid oscillator version of the bound state approach to strange baryons proposed by Kaplan and Klebanov [9] and used later in [10]. This model has definite advantages before collective coordinates quantization method when heavy flavors are included

\footnotetext{
${ }^{1}$ The work supported in part by the Russian Fund for Fundamental Research, grant 95-02-03868a and presented at the Workshop on Science at Japan Hadron Facility (JHF98), KEK, Tsukuba, March 4-7, 1998
} 
into consideration, first of all, because of its simplicity. However, some apparent drawbacks are present also.

\section{Rigid oscillator model}

The ansatz for the chiral fields used in [9, 10] is:

$$
U(r, t)=R(t) U_{0}(r) R^{\dagger}(t), \quad R(t)=A(t) S(t),
$$

where $U_{0}$ is $S U(2)$ soliton embedded into $S U(3)$ in usual way (into left upper corner), $A(t) \in S U(2)$ describes $S U(2)$ rotations, $S(t) \in S U(3)$ describes rotations in the "charm" or "bottom" direction. For definiteness we shall consider the extension of the $(u, d)$ $S U$ (2) Skyrme model in charmed direction, when $D$ is the field of $D$-mesons. But it is clear that quite similar the extension can be made in bottom and top direction.

$$
S(t)=\exp (i D(t)), \quad D(t)=\sum_{a=4, \ldots 7} D_{a}(t) \lambda_{a}
$$

$\lambda_{a}$ are Gell-Mann matrices of $(u, d, c)$ or $(u, d, b) S U(3)$ groups. The $(u, d, b) S U(3)$ subgroup is quite analogous to the $(u, d, s)$ one, for $(u, d, c)$ subgroup simple redefiniton of hypercharge should be made. $D_{4}=\left(D^{0}+\bar{D}^{0}\right) / \sqrt{2}, D_{5}=i\left(D^{0}-\bar{D}^{0}\right) / \sqrt{2}$, etc.

After some calculation the well known Lagrangian of the Skyrme model in the lowest order in field $D$ takes the form [9, 10]:

$$
L=-M_{c l, B}+4 \Theta_{F, B} \dot{D}^{\dagger} \dot{D}-\Gamma_{B}\left(m_{D}^{2}-m_{\pi}^{2}\right) D^{\dagger} D+i \frac{N_{c} B}{2}\left(D^{\dagger} \dot{D}-\dot{D}^{\dagger} D\right) .
$$

Here $D$ is a doublet formed by $D^{0}$ and $D^{-}$mesons, and we maintained our former notation for the moment of inertia for the rotation into "strange", "charm" or "bottom" direction $\Theta_{c}=\Theta_{b}=\Theta_{s}=\Theta_{F}$. This moment of inertia has simple analytical form for arbitrary starting $S U(2)$ skyrmion, regardless its symmetry properties:

$$
\Theta_{F, B}=\frac{1}{8} \int\left(1-c_{f}\right)\left[F_{\pi}^{2}+\frac{1}{e^{2}}\left((\overrightarrow{d f})^{2}+s_{f}^{2}(\vec{d} \alpha)^{2}+s_{f}^{2} s_{\alpha}^{2}(\vec{d} \beta)^{2}\right)\right] d^{3} r
$$

$F_{\pi}$ and $e$ are the parameters of the model. The general parametrization of the $S U(2)$ skyrmions has been used here, $U=c_{f}+s_{f} \vec{\tau} \vec{n}$ with $n_{z}=c_{\alpha}, n_{x}=s_{\alpha} c_{\beta}, n_{y}=s_{\alpha} s_{\beta}$, $s_{f}=\sin f, c_{f}=\cos f$, etc. For the axially symmetrical ansatz $\beta=n \phi, \phi$ is the azimuthal angle, and $\Theta_{F, B}$ takes the form drawn in [1]]:

$$
\Theta_{F, B}=\frac{\pi}{4} \int\left(1-c_{f}\right)\left[F_{\pi}^{2}+\frac{1}{e^{2}}\left((f, f)+s_{f}^{2}(\alpha, \alpha)+\frac{n^{2}}{r^{2}} s_{f}^{2} s_{\alpha}^{2}\right)\right] r d r d z=\frac{\Gamma_{B}}{4}+\Theta_{F, B}^{S k},
$$

$(f, f)=(\partial f / \partial r)^{2}+(\partial f / \partial z)^{2}, r$ and $z$ being cylindrical coordinates. The quantity $\Gamma_{B}$ defines the contribution of the mass term in the Lagrangian:

$$
\Gamma_{B}=\frac{F_{\pi}^{2}}{2} \int\left(1-c_{f}\right) d^{3} r
$$

Numerical values of $\Theta_{F, B}, \Gamma_{B}$ and some other quantities are shown in Table 1 below. 
The term in (3) proportional to $N_{c} B$ appears from the Wess-Zumino-Witten term in the action and is responsible, within this approach, for the splitting between excitation energies of charm and anticharm (flavor and antiflavor in general case) 8]- [10]. $N_{c}$ is the number of colors in the underlying $Q C D$, in all other cases here the index $c$ means the charm quantum number. $B$ is the baryon number of the configuration which can be written in terms of the functions $f, \alpha$ and $\beta$ as

$$
B=-\frac{1}{2 \pi^{2}} \int s_{f}^{2} s_{\alpha}(\vec{\partial} f \vec{\partial} \alpha \vec{\partial} \beta) d^{3} r
$$

In other words, it is the Wronskian of the system described by 3 profiles, $f, \alpha$ and $\beta$ [2]. For the axially symmetrical configuration possessing also symmetry $z \rightarrow-z$, $B=n(f(0)-f(\infty)) / \pi=n$ for configurations of lowest energy.

As it was noted in [10] deviations of the field $D$ from the vacuum decrease with increasing mass $m_{D}$, as well as with increasing number of colors $N_{c}$, and the method works at any $m_{D}$ - for bottom and even top quantum number also. The zero modes quantum corrections due to rotation with the matrix $A(t)$ have the order of magnitude $N_{c}^{-1}$ and are not crucial but also important (see also section 4 ).

\section{Flavor excitation frequences}

After the canonical quantization procedure the Hamiltonian of the system takes the form:

$$
H_{B}=M_{c l, B}+\frac{1}{4 \Theta_{F, B}} \Pi^{\dagger} \Pi+\left(\Gamma_{B} \bar{m}_{D}^{2}+\frac{N_{c}^{2} B^{2}}{16 \Theta_{F, B}}\right) D^{\dagger} D-i \frac{N_{c} B}{8 \Theta_{F, B}}\left(D^{\dagger} \Pi-\Pi^{\dagger} D\right) .
$$

$\bar{m}_{D}^{2}=m_{D}^{2}-m_{\pi}^{2}$ The momentum $\Pi$ is canonically conjugate to variable $D$. Eq. (7) describes the oscillator-type motion of the field $D$ in the background formed by the $(u, d) S U(2)$ soliton. After the diagonalization which can be done explicitely according to [9, 10] the Hamiltonian can be written as

$$
H_{B}=M_{c l, B}+\omega_{F, B} a^{\dagger} a+\bar{\omega}_{F, B} b^{\dagger} b+O\left(1 / N_{c}\right)
$$

with $a^{\dagger}, b^{\dagger}$ being the operators of creation of charm and anticharm (bottom and antibottom) quantum number, $\omega_{F, B}$ and $\bar{\omega}_{F, B}$ being the frequences of heavy flavor (antiflavor) excitation. $D$ and $\Pi$ are connected with $a$ and $b$ in the following way [9, 10]:

$$
D^{i}=\frac{1}{\sqrt{N_{c} B \mu_{F, B}}}\left(a^{i}+b^{\dagger i}\right), \quad \Pi^{i}=\frac{\sqrt{N_{c} B \mu_{F, B}}}{2 i}\left(a^{i}-b^{\dagger i}\right)
$$

with

$$
\mu_{F, B}=\left(1+16 \bar{m}_{D}^{2} \Gamma_{B} \Theta_{F, B} /\left(N_{c} B\right)^{2}\right)^{1 / 2} .
$$

For the lowest states the values of $D$ are small,

$$
D \sim\left[4 \Gamma_{B} \Theta_{F, B} \bar{m}_{D}^{2}+N_{c}^{2} B^{2} / 4\right]^{-1 / 4} .
$$

The flavor (antiflavor) excitation frequences $\omega$ and $\bar{\omega}$ are:

$$
\omega_{F, B}=\frac{N_{c} B}{8 \Theta_{F, B}}\left(\mu_{F, B}-1\right), \quad \bar{\omega}_{F, B}=\frac{N_{c} B}{8 \Theta_{F, B}}\left(\mu_{F, B}+1\right)
$$


It should be noted that the difference $\bar{\omega}_{F, B}-\omega_{F, B}=N_{c} B /\left(4 \Theta_{F, B}\right)$ coincides in the leading order in $N_{c}$ with that obtained in the collective coordinates approach [12, 13]. Indeed, in the collective coordinates approach the zero-modes energy of the soliton rotated in the $S U(3)$ configuration space and depending on the "flavor" inertia $\Theta_{F, B}$ can be written as:

$$
E_{\text {rot }}\left(\Theta_{F, B}\right)=\frac{1}{4 \Theta_{F, B}}\left[N_{c} B+n_{q \bar{q}}\left(N_{c} B+2 n_{q \bar{q}}+2-2 T_{r}\right)\right]
$$

where $n_{q \bar{q}}$ is the number of additional quark-antiquark pairs present in the quantized state, $N_{c} B+3 n_{q \bar{q}}=p+2 q, p, q$ are the numbers of indices in the spinor describing the $S U$ (3) irrep, $T_{r}=\left(p+n_{q \bar{q}}\right) / 2$ is the so called right isospin characterizing irrep (see [13] where the $B=1, n_{q \bar{q}}=0$ case was considered, and [12] where (11) was obtained for $\left.N_{c}=3\right)$. The term proportional to $n_{q \bar{q}} N_{c} B$ in (11) coincides with the difference of $\bar{\omega}_{F, B}-\omega_{F, B}$ in (10).

For the difference of the frequences of excitation in cases of $B \geq 2$ and $B=1$ systems we obtain:

$$
\Delta \omega \simeq \frac{\bar{m}_{F}}{2}\left[\left(\frac{\Gamma_{1}}{\Theta_{F, 1}}\right)^{1 / 2}-\left(\frac{\Gamma_{B}}{\Theta_{F, B}}\right)^{1 / 2}\right]
$$

It is proportional to the heavy quark mass $m_{F}$ and is positive if $\Gamma_{1} / \Theta_{F, 1} \geq \Gamma_{B} / \Theta_{F, B}$. For $B=2,3$ it is really so. The characteristics of $S U(2)$ toroidal solitons with baryon numbers $B=2,3$ and 4 have been calculated previously [14]. For $B=2$ they coincide with good accuracy with those given later in [10]. For greater baryon numbers some configurations of lower energy have been found [15, 16], but necessary quantities like $\Theta_{F, B}$ and $\Gamma_{B}$ are absent, still.

As a result, the binding energy of heavy flavored dibaryons, tribaryons, etc. increases in comparison with strange flavor case, as it can be seen from the results of numerical estimates shown in Table 1.

\section{$41 / N_{c}$ zero modes corrections and binding energies estimates}

The $\sim 1 / N_{c}$ zero modes quantum correction to the energies of $B S$ can be estimated according to the expression [9, 10]:

$$
\Delta E_{1 / N_{c}}=\frac{1}{2 \Theta_{T, B}}\left[c_{F, B} T_{r}\left(T_{r}+1\right)+\left(1-c_{F, B}\right) I(I+1)+\left(\bar{c}_{F, B}-c_{F, B}\right) T(T+1)\right],
$$

where $I$ is the isospin of the $B S, T_{r}$ is the quantity analogous to the "right" isospin $T_{r}$ in the collective coordinates approach [3, 11, 6], and $\vec{T}_{r}=\vec{I}^{b f}+\vec{T}$.

$$
c_{F, B}=1-\frac{\Theta_{T, B}}{2 \Theta_{F, B} \mu_{F, B}}\left(\mu_{F, B}-1\right), \quad \bar{c}_{F, B}=1-\frac{\Theta_{T, B}}{\Theta_{F, B}\left(\mu_{F, B}\right)^{2}}\left(\mu_{F, B}-1\right) .
$$

In the rigid oscillator model the states predicted are not identified with definite $S U(3)$ or $S U(4)$ representations. However, it can be done, as it was shown in [10]. The quantization condition $(p+2 q) / 3=B$ [3] for arbitrary $N_{c}$ is changed to $(p+2 q)=$ $N_{c} B+3 n_{q \bar{q}}$. For example, the state with $c=2, I=0$ and $n_{q \bar{q}}=0$ should belong to the 27 - plet of $(u, d, c) S U(3)$ group, if $N_{c}=3$, see also [10]. For 27-plet of dibaryons $T_{r}=1$, 
for antidecuplet $T_{r}=0$. For 35 -plet of tribaryons $T_{r}=1 / 2$, for arbitrary $(p, q)$ irrep which the $B S$ belongs to $T_{r}=p / 2$ if $n_{q \bar{q}}=0 . I$ and $T$ take the lowest possible values, 0 or $1 / 2$ in our case. If $\Theta_{F} \rightarrow \infty$ Eq. (13) goes over into the expression obtained for axially symmetrical $B S$ in collective coordinate approach [11], in realistic case with $\Theta_{T} / \Theta_{F} \simeq 2.7$ the structure of (13) is more complicated.

The quantum correction due to usual space rotations, also of the order of $1 / N_{c}$ is exactly of the same form as obtained in [11], see [9, 10]. The binding energies shown in Table 1 are defined relative to the decay into $B$ baryons, nucleons or flavored hyperons. The binding energy, e.g. of $B=4$ state relative to 2 dibaryons will be smaller or negative. Since we are interested in the lowest energy states we discuss here the baryonic systems with the lowest allowed angular momentum, $J=0$ for $B=2,4$, and $J=3 / 2$ for $B=3$. The latter value is due to the constraint because of symmetry properties of the configuration. The value $J=1 / 2$ is allowed for the configuration found in [15].

For $B=3$ and 4 toroidal configurations we used here do not correspond to the minimum of static energy, but only for such configurations the necessary quantities, $\Theta_{F, B}, \Gamma_{B}$ are known. For $B=3$ the toroidal configuration does not differ much in energy from the tetrahedral one which is known to be the configuration of minimal energy [15, 16]. (The masses of stranglets obtained from bound skyrmions with $B$ up to 17 [16 have been estimated recently in [17] in the bound state soliton model.) For $B=4$ the difference is large, $\sim 300 \mathrm{Mev}$ in energy. However, it would be incorrect to decrease all $B=4$ energies by $300 \mathrm{Mev}$ and increase the binding energies, because other characteristics of solitons and, therefore, the excitation energies $\omega_{c}$ and $\omega_{b}$ also change. Some reasonable extrapolation for $B=4$ is shown in Table 1 .

\begin{tabular}{|l|l|l|l|l|l|l|l|l|l|l|l|l|l|}
\hline$B$ & $M_{c l, B}$ & $\Theta_{F, B}$ & $\Theta_{T, B}$ & $\Theta_{J, B}$ & $\Gamma_{B}$ & $\omega_{s}$ & $\omega_{c}$ & $\omega_{b}$ & $\epsilon_{s=-2}$ & $\epsilon_{c=1}$ & $\epsilon_{c=2}$ & $\epsilon_{b=-1}$ & $\epsilon_{b=-2}$ \\
\hline 1 & 0.865 & 1.86 & 5.14 & 5.14 & 3.98 & 0.200 & 1.18 & 3.66 & - & - & - & - & - \\
\hline 2 & 1.656 & 3.79 & 10.55 & 16.45 & 7.80 & 0.196 & 1.15 & 3.62 & 0.096 & 0.16 & 0.15 & 0.18 & 0.19 \\
\hline 3 & 2.523 & 6.16 & 16.85 & 37.85 & 12.85 & 0.205 & 1.17 & 3.63 & 0.12 & 0.22 & 0.23 & 0.27 & 0.27 \\
\hline 4 & 3.446 & 8.84 & 23.65 & 72.5 & 18.80 & 0.215 & 1.19 & 3.68 & 0.18 & 0.23 & 0.21 & 0.25 & 0.25 \\
\hline $4^{*}$ & 3.140 & - & - & - & - & 0.196 & 1.15 & 3.62 & 0.52 & 0.58 & 0.61 & 0.60 & 0.65 \\
\hline
\end{tabular}

Table 1. The static characteristics of the $B=1$ hedgehog and toroidal solitons with $B=2,3,4$ [14]: $M_{c l, B}$ in Gev, moments of inertia $\Theta_{F, B}=\Theta_{c}=\Theta_{b}, \Theta_{T}, \Theta_{J}$ and $\Gamma$ in $G e v^{-1}$. The excitation frequences $\omega_{s, c, b}$ - in Gev. The binding energies (in Gev) of baryonic systems with $B=2,3,4, s=-2\left(\epsilon_{s=-2}\right)$, charm $c=1,2\left(\epsilon_{c=1,2}\right)$ and bottom $b=-1,-2\left(\epsilon_{b=-1,-2}\right)$ are shown. The parameters of the model $F_{\pi}=108 \mathrm{Mev}, e=4.84\left[3\right.$. The line $B=4^{*}$ shows the binding energies for $B=4$ configuration found in 15, 16] with extrapolation $\omega_{B=4}=\omega_{B=2}$. The uncertainty of these estimates within our choice of the model and configurations is $\sim 0.02 G e v$.

The binding energy of the deuteron-like state within this approach is 0.16 Gev, the binding energy of the $N N$ scattering atate with isospin $T=1$ is $0.127 \mathrm{Gev}$. If we assume that the nonzero modes quantum corrections - due to vibration and breathing modes, as well as 1-loop corrections - are approximately the same for all states with the same $B$ then we should renormalize the energy of each state obtained in this way, i.e. we should subtract just $0.127 \mathrm{Gev}$ from the binding energies shown in Table 1 (the energy of the virtual level, $0.067 \mathrm{Mev}$, is negligible, of course). 
Renormalization of this type was done also previously in [11]. It is clear that after this renormalization the state with strangeness $S=-2$ becomes unbound, $\sim 0.03 \mathrm{Gev}$ above the threshold, but the states with charm or bottom remain bound. These estimates are crude, of course, because the binding energy of the deuteron is about $\sim 0.03 \mathrm{Gev}$ within the same approach. However, they show clearly that $B S$ with charm and bottom have more chances to be bound relative to strong interactions than strange $B S$.

\section{$5 \quad$ Symmetry breaking in flavor decay constants}

The estimates of binding energy were made in [9, 10] with the constant $F_{K}=F_{\pi}$, and similar assumption was made above to get the results shown in Table 1 . However, symmetry breaking takes place not only in the masses of baryons, but also in decay constants, since generally $F_{F}$ is different from $F_{\pi}$. As it was shown by Riska and Scoccola [18], the large enough values of $F_{F}$ allow to remove the apparent overbinding of heavy mesons by $S U(2)$ solitons which is characteristic for the bound state models with $F_{F}=F_{\pi}$.

For kaons, $F_{K}=1.22 F_{\pi}$, and this is important for the description of mass differences inside the octet and decuplet of baryons. Therefore, it seems necessary to investigate the binding energies of $B S$ also for the case of this kind of flavor symmetry breaking (FSB).

The mass term in the Lagrangian (3) should be changed to

$$
L_{M}=-\left(m_{F}^{2} F_{F}^{2} / F_{\pi}^{2}-m_{\pi}^{2}\right) \Gamma_{B} .
$$

It is assumed that the pion mass term is included into the classical mass of the soliton $M_{c l}$. Corresponding change should be made in the Hamiltonian (7) also. The second order term in the Lagrangian should be modified also, but the corresponding contribution to the mass or energy is much smaller than that given by (15) because it is proportional to cosf in the integrand and cancellations take place in the integration over $d^{3} r$, see [11, 20].

It is not difficult to calculate the "flavor" moment of inertia in the case of $F S B$ :

$$
\Theta_{F, B} \rightarrow \Theta_{F, B}+\left(F_{F}^{2} / F_{\pi}^{2}-1\right) \Gamma_{B} / 4
$$

The modified inertia $\Theta_{F}$ can be calculated easily according to (16) for any $F_{F}$ since $\Theta_{F}$ and $\Gamma_{B}$ are known.

The following expression can be obtained for the excitation energy:

$$
\omega_{F, B} \simeq \frac{m_{F}}{\left[1+4 \Theta_{F, B}^{S k} /\left(r_{F}^{2} \Gamma_{B}\right)\right]^{1 / 2}}-\frac{N_{c} B}{8 \Theta_{F, B}}
$$

Here $r_{F}=F_{F} / F_{\pi}, \Theta_{F, B}^{S k}$ is the Skyrme term contribution to the moment of inertia, so we can write:

$$
\Theta_{F, B}=\Theta_{F, B}^{S k}+r_{F}^{2} \Gamma_{B} / 4
$$

For our choice of the model parameters $\Theta_{F, B}^{S k}$ is a bit smaller than $\Gamma_{B} / 4$. 


\begin{tabular}{|l|l|l|l|l|l|l|l|l|l|l|l|l|}
\hline$B$ & $\Theta_{s, B}$ & $\Theta_{c, B}$ & $\Theta_{b, B}$ & $\omega_{s}$ & $\omega_{c}$ & $\omega_{b}$ & $\bar{c}_{s, B}$ & $\epsilon_{s=-2}$ & $\bar{c}_{c, B}$ & $\epsilon_{c=2}$ & $\bar{c}_{b, B}$ & $\epsilon_{b=-2}$ \\
\hline 1 & 2.346 & 3.104 & 4.845 & 0.255 & 1.466 & 4.708 & 0.480 & - & 0.883 & - & 0.980 & - \\
\hline 2 & 4.742 & 6.228 & 9.640 & 0.250 & 1.447 & 4.671 & 0.472 & 0.11 & 0.880 & 0.16 & 0.982 & 0.20 \\
\hline 3 & 7.729 & 10.18 & 15.80 & 0.260 & 1.464 & 4.684 & 0.498 & 0.15 & 0.892 & 0.22 & 0.984 & 0.28 \\
\hline 4 & 11.13 & 14.71 & 22.94 & 0.270 & 1.481 & 4.713 & 0.528 & 0.20 & 0.903 & 0.26 & 0.986 & 0.30 \\
\hline $4^{*}$ & - & - & - & 0.250 & 1.447 & 4.671 & - & 0.52 & - & 0.60 & - & 0.66 \\
\hline
\end{tabular}

Table 2. The flavor inertia $\Theta_{F, B}$ (in $G e v$ ) for $F_{K} / F_{\pi}=1.22, F_{c} / F_{\pi}=1.5, F_{b} / F_{\pi}=2$. Excitation frequences and some binding energies for $|F|=2$ baryonic systems with flavor symmetry breaking in the constants $F_{F}$ - in Gev. The quantities $\bar{c}_{F, B}$ defining the suppression of zero-modes quantum corrections are shown also.

We took here $r_{c}=F_{D} / F_{\pi}=1.5$ and $r_{b}=F_{b} / F_{\pi}=2$ because these values of decay constants ratios allow to get the masses of lowest baryons $\Lambda_{c}$ and $\Lambda_{b}$ not very far from the observed values. $r_{c}$ does not contradict to the experimental restriction. As it can be seen from Table 2 the flavor symmetry violation in meson decay constants leads to moderate increase of the binding energies of $B S$ with different flavors, and the tendency of increase of $\epsilon_{F}$ with increasing mass of flavor becomes more striking.

It is of interest to look at the case of large $F S B$, when the ratio $r_{F}=F_{F} / F_{\pi} \gg 1$. In this case it is easy to find out what is the effective potential for the heavy meson in the $S U(2)$ soliton background. The following expression can be obtained:

$$
\omega_{F, B} \simeq m_{F}\left[1-2 \Theta_{F, B}^{S k} /\left(\Gamma_{B} r_{F}^{2}\right)\right]-m_{\pi}^{2} /\left(2 m_{F} r_{F}^{2}\right)-N_{c} B /\left(2 \Gamma_{B} r_{F}^{2}\right)
$$

This defines the average potential of the heavy meson bound by $S U(2)$ soliton. Obviously, $\omega_{F, B} \rightarrow m_{F}$ when $r_{F} \rightarrow \infty$.

\section{Conclusions}

To conclude, we estimated the binding energies of dibaryons, tribaryons and tetrabaryons with nonzero charm and bottom. For the top quantum number similar results can be obtained, but the spectroscopy of mesons, baryons and baryonic systems with t-number will not be available, probably, because of the large width of the top quark.

For $r_{t}=F_{t} / F_{\pi}=2$ the $\omega_{t}-s$ are about $157-158 \mathrm{Gev}$ and the binding energies $\sim 1 \mathrm{Gev}$. To obtain the excitation energies $\omega \simeq m_{t}=175 \mathrm{Gev}$ and the binding energies of the same order as we have, e.g. for strange $B S$, we should take $r_{t} \sim \sqrt{m_{t}}$. For example, for $r_{t}=17 \omega \simeq 174.7 \mathrm{Gev}$ and $\epsilon_{t=2} \simeq 0.2 \mathrm{Gev}$. However, such big value of $r_{t}$ seems to be unrealistic. Anyway, it may be of interest that for very large mass of the "flavored" meson the scale of the binding energies of $B S$ is connected with the scale of the ratio $F_{F} / F_{\pi}$.

Since the binding energies for strangeness, charm or bottom increase with increasing mass of the flavor for realistic values of constant $F$, the charmed and bottomed baryonic systems have more chances to be bound than strange $B S$. This is in agreement with the experimental fact that the difference of masses of $\Lambda_{F}$ baryons and corresponding pseudoscalar mesons, $P_{F}(K, D$ or $B)$ decreases with increasing 
mass of flavor, just indicating that the binding energy of flavored quark in the baryon increases in comparison with that in meson with increasing $m_{F}$. Nonzero quantum corrections to the energy of charmed (bottomed) baryonic systems are expected to be smaller in comparison with strange baryonic systems, because of the greater mass of charmed (bottomed) quarks or mesons.

The apparent drawback of the approach exploited in the present paper is that the motion of the system into the "charm" or "bottom" direction is considered independently from other motions. Consideration of the $B S$ with "mixed" flavors is possible, in principle, but it demands more complicated treatment.

The collective coordinates approach with the rigid or soft rotator variant of the model usually gives the masses of baryons considerably greater than the KaplanKlebanov-Westerberg model we used here, if the Casimir energies are not taken into account [3, 11, 18]. One of the sources of this difference is the presence of the zeromodes contribution in the rotation energy of the order of $N_{c} / \Theta_{F}$, see (11) [13, 11, 18], which is absent in the oscillator model. As it was shown recently by Walliser for the $B=1$ sector [13] this large contribution is cancelled almost completely by the 1-loop correction - zero-point Casimir energy which is of the same order of magnitude, $N_{c}^{0}$ [19]. Anyway, since both approaches have led to similar results in the case of strange baryonic systems, we may expect the same for the case of charmlets and bottomlets, so, our results should be valid qualitatively, at least.

The threshold for the charm production on a free nucleon is about $12 \mathrm{Gev}$, for double charm - about 25.2Gev. However, for nuclei as a target the thresholds are much lower due to two-step processes with mesons in intermediate states and due to normal Fermi-motion of nucleons inside the target nucleus (see, e.g. [22]). Therefore, production of states with $c=1$ and even $c=2$ will be available on accelerators like future Japan Hadron Facility (energy 50Gev), the subthreshold production of $b=-1$ systems on nuclei with small probability also will be possible.

When the present paper was almost completed I have found a paper [23] where the charmed few baryon systems have been considered within more conventional potential approach. The $c=1$ system with $B=3$ was found to be not bound, probably (the binding energy is of the order of $1 \mathrm{Mev}$ ), The $B=4$ system was found to be bound with the binding energy not exceeding $\sim 10 \mathrm{Mev}$.

I am indebted to H.Walliser for useful discussions of the skyrmions quantization at arbitrary $N_{c}$ and $N_{F}$, and for valuable remarks. I appreciate also critical remarks by A.Penin.

\section{References}

1. J.K. Ahn et al, KEK PS E224 Collaboration, AIP Conf. Proc. 412, 923-926 (1997)

2. L.D. Faddeev, V.E. Korepin, Phys.Rept. 42,1 (1978)

3. G.S. Adkins, C.R. Nappi, E. Witten, Nucl.Phys. B228,552 (1983); G.S. Adkins, C.R. Nappi, Nucl.Phys. B233, 109 (1984); G. Guadagnini, Nucl.Phys. B236, 35 (1984)

4. J.T. Reed et al, Phys. Rev. 168, 1495 (1968)

5. B. Sechi-Zorn et al, Phys.Rev. 175, 1452 (1968); G. Alexander et al, Phys.Rev. 173, 1452 (1968)

6. V.B. Kopeliovich, Genshikaku Kenkyu 41, 171 (1997); hep-ph/9712453, Nucl. Phys. A, in print; JETP 85, 1060(1997) [ZhETF 112, 1941 (1997)]; Phys. Atom. Nucl. 56, 1084 (1993)

7. C.G. Callan, I.R. Klebanov, Nucl. Phys. B262,365 (1985) 
8. N. Scoccola, H. Nadeau, M. Nowak, M. Rho, Phys. Lett. B201, 425 (1988); C.G. Callan, K. Hornbostel, I.R. Klebanov, Phys.Lett. B202, 269 (1988); J.P. Blaizot, M. Rho, N. Scoccola, ibid. 209, 27 (1988); N. Scoccola, D.P. Min, H. Nadeau, M. Rho, Nucl. Phys. A505, 497 (1989)

9. D. Kaplan, I.R. Klebanov, Nucl.Phys. B335,45 (1990)

10. K.M. Westerberg, I.R. Klebanov, Phys. Rev. D50, 5834 (1994); I.R. Klebanov, K.M. Westerberg, Phys.Rev. D53, 2804 (1996)

11. V.B. Kopeliovich, B. Schwesinger, B.E. Stern, Nucl.Phys. A549,485 (1992); V.B. Kopeliovich, Yad.Fiz. 47,1495 (1988); Yad.Fiz. 51, 241 (1990)

12. V.B. Kopeliovich, Phys.Lett. 259B, 234 (1991)

13. H. Walliser, hep-ph/9710232

14. V.B. Kopeliovich, B.E. Stern, NORDITA. Copenhagen-NORDITA-89-34 (89, REC.SEP); JETP Lett. 45,203 (1987) [Pis'ma v ZhETF, 45,165 (1987)]

15. E. Braaten, S. Townsend, L. Carson, Phys.Lett. B235,147 (1990)

16. R.A. Battye, P.M. Sutcliffe, Phys.Lett. B391, 150 (1997); Phys.Rev.Lett. 79, 363 (1997)

17. M. Schvellinger, N.N. Scoccola, hep-ph/9801347

18. D.O. Riska, N.N. Scoccola, Phys.Lett. B265, 188 (1991)

19. M. Bjornberg, K. Dannbom, D.O. Riska, N.N. Scoccola, Nucl.Phys. A539, 662 (1992)

20. H. Walliser, Nucl.Phys. A548,649 (1992)

21. B. Moussalam, Ann. of Phys. (N.Y.) 225,264 (1993); F. Meier, H. Walliser, Phys. Rept. 289,383 (1997)

22. V.B. Kopeliovich, Phys.Rept. 139, 51 (1986); Yad.Fiz. 42, 854 (1985)

23. B.F. Gibson, C.B. Dover, G. Bhamathi, D.R. Lehman, Phys.Rev. C27, 2085 (1983) 